\title{
La diversidad floral del borde afecta la riqueza y abundancia de visitantes florales nativos en cultivos de girasol
}

\author{
Agustín Sáez ${ }^{\varpi}$; Malena Sabatino \& Marcelo Aizen \\ Laboratorio Ecotono-CRUB, Universidad Nacional del Comahue-INIBIOMA. San Carlos de Bariloche, Río Negro, Argentina.
}

\begin{abstract}
Resumen. La declinación en la diversidad y abundancia de polinizadores relacionada a diversos tipos de disturbios de origen antrópico es un tema de debate en el contexto del cambio en el uso de la tierra. Dentro de este debate se ha enfatizado la importancia de los remanentes de hábitats naturales y semi-naturales como reservorios de polinizadores para cultivos dependientes de ellos. El sudeste de la Provincia de Buenos Aires constituye una zona agrícolo-ganadera con un alto grado de uso y alteración de la tierra. Sin embargo, los márgenes de cultivos asociados a los bordes de caminos pueden proveer recursos florísticos y sitios de nidificación para una gran diversidad de polinizadores, los que podrían, eventualmente, incrementar la polinización y la productividad de muchos cultivos aledaños dependientes de ellos. Aquí evaluamos el efecto de la diversidad floral de los bordes de cultivos sobre la riqueza y abundancia de los visitantes florales en 17 lotes de girasol en la zona de Balcarce y Lobería. Nuestros resultados indican que la diversidad floral del borde tuvo un efecto positivo sobre la riqueza y abundancia de visitantes florales nativos en el cultivo adyacente. En consecuencia, los servicios de polinización en estos agroecosistemas podrían ser promovidos por un incremento en la cantidad y diversidad de estos hábitats marginales.
\end{abstract}

[Palabras clave: visitantes florales, diversidad floral, agroecosistemas, girasol]

\begin{abstract}
AвsтRACт. The floral diversity of field margins influences the richness and abundance of native flower visitors in sunflower fields: Pollinator decline has been related to different types of anthropogenic disturbances, a topic largely debated in the context of global change. Within this debate, it has been stressed the importance of natural or semi-natural habitat remnants as pollinator sources for nearby agricultural fields. The SW of Buenos Aires Province is a highly-impacted and intensively-used agricultural region. However, field margins associated with country roads can provide diverse floristic resources and nesting sites for a variety of pollinators, which can eventually improve pollination and productivity of neighboring pollinator-dependent crops. Here, we evaluated the effect of floral diversity of field margins on the richness and abundance of floral visitors across 17 sunflower lots in the districts of Balcarce and Lobería. Our results indicate that floral diversity of field margins had a positive effect on the richness and abundance of native floral visitors in the adjacent crop field. As a consequence, pollination services in these agroecosystems would be promoted by increasing the amount and diversity of these marginal habitats.
\end{abstract}

[Keywords: floral visitors, floral diversity, agro-ecosystems, sunflower]

\section{INTRODUCCIÓN}

La polinización mediada por animales es uno de los procesos clave que garantiza la reproducción de las plantas con flor, y es un factor limitante en la cantidad de semillas producidas (Klein et al. 2007; NRC 2007; Ollerton et al. 2011). Este proceso no sólo representa un factor fundamental en el mantenimiento de la diversidad de las angiospermas sino que también cumple un rol importante en la producción global de alimentos. Aproximadamente, el $70 \%$ de las plantas cultivadas dependen en diferente medida de la presencia de polinizadores efectivos, en su mayoría abejas, para maximizar su producción (Klein et al. 2007). Diversos estudios han demostrado que la polinización de muchos de estos cultivos se ve incrementada por la presencia de

Editora asociada: Adriana Salvo

$\bowtie$ agustinsaez@live.com.ar polinizadores silvestres que dependen en su mayoría de hábitats naturales o seminaturales para nidificar y obtener recursos florales (Klein et al. 2003a y b; Kremen et al. 2002, 2004; Ricketts 2004; Greenleaf \& Kremen 2006a y b; Chacoff et al. 2008). Sin embargo, el uso excesivo e inadecuado de pesticidas y herbicidas, la introducción de insectos no nativos y, principalmente, la destrucción y fragmentación del hábitat original ha causado una declinación en la abundancia y diversidad de los polinizadores en varias regiones del mundo (Sala et al. 2000; Ricketts et al. 2008; Winfree et al. 2009; Potts et al. 2010).

El servicio de polinización en ambientes agrícolas es provisto principalmente por poblaciones domésticas y silvestres de la abeja melífera (Apis mellifera). Sin embargo, los insectos silvestres, incluso en baja densidad, 
cumplen un rol aún más importante que el de $A$. mellifera en la formación de frutos y/o semillas de casi todas las especies cultivadas que dependen de la polinización animal (Garibaldi et al. 2013). Por este motivo, los ensambles de polinizadores diversos son críticos para sostener altos rendimientos agrícolas. La mayoría de estos polinizadores silvestres nidifican en ambientes naturales o semi-naturales y utilizan el néctar y/o polen que ofrecen los cultivos que se encuentran dentro de su rango de forrajeo (Ricketts 2004). Este rango es particular de cada especie y determina la escala espacial en la cual estos polinizadores pueden tener un rol en la polinización de los cultivos aledaños (Bronstein 1995; Kremen 2005; Greenleaf et al. 2007). Sin embargo, los recursos alimenticios brindados por los cultivos son localmente abundantes y temporalmente restringidos, por lo que los ambientes naturales y semi-naturales permitirían la continuidad espacio-temporal en la provisión de recursos alimenticios y la supervivencia de los polinizadores en los agroecosistemas.

La relevancia de los remanentes de hábitats naturales y semi-naturales en el paisaje agrícola ha sido motivo de debate y estudio ya que, más allá de su limitada extensión, son de gran importancia como reservorios de biodiversidad (Hendrickx et al. 2007; Billeter et al. 2008) y como proveedores de diferentes servicios ecosistémicos (Costanza et al. 1997; Kremen et al. 2004; Klein et al. 2007; Carvalheiro et al. 2011). Sin embargo, como ningún ensamble existe aislado en una comunidad, su diversidad y abundancia pueden depender en gran medida de la diversidad de los recursos provistos por el nivel trófico inferior (Altieri 1999; Clough et al. 2007). Para el caso específico de los polinizadores, esperamos que su abundancia y riqueza estén relacionados con la diversidad floral, particularmente de plantas entomófilas, de los hábitats donde se encuentran, a través de dos procesos principales: uno relacionado a la amplitud de la ventana fenológica (i.e., duración total del período de floración), y el otro a la complementariedad y suplementariedad de recursos. Por un lado, es de esperar que comunidades florísticas más diversas presenten una mayor amplitud de la ventana fenológica, dado que distintas especies vegetales poseen épocas de floración características, que tienden a diferir entre ellas (Kudo 2006). Una mayor amplitud fenológica permitiría el sustento de poblaciones de un mayor número de especies de polinizadores debido a que diferentes polinizadores presentan picos de actividad temporal distintos y muchos permanecen activos durante períodos más extensos que el de la floración de una o pocas especies de plantas (Olesen et al. 2008). Por otro lado, es de esperar que comunidades florísticas más diversas estén asociadas a la oferta de una mayor diversidad de recursos (e.g., néctar, polen) (Ghazoul 2006). La dicotomía más aparente es la que existe entre especies de plantas que ofrecen principalmente néctar y aquellas que ofrecen principalmente polen como recompensa alimenticia. Si bien algunos polinizadores consumen exclusivamente uno $\mathrm{u}$ otro recurso floral (e.g., los picaflores sólo consumen néctar), ambos son imprescindibles para la subsistencia de la mayoría de las especies de abejas, que es el grupo de polinizadores más importante. Además de recursos alimenticios, las plantas también ofrecen refugio, así como sitios de nidificación y apareamiento, que son de gran importancia para el mantenimiento de distintas especies de polinizadores. En consecuencia, podemos predecir que los ambientes con una mayor diversidad floral albergan ensambles de polinizadores más abundantes y de mayor riqueza, los cuales podrían brindar un servicio de polinización de mejor calidad a los cultivos aledaños (Garibaldi et al. 2013).

El sudeste de Provincia de Buenos Aires es una zona agrícola-ganadera en donde las actividades agropecuarias desarrolladas principalmente durante los últimos 80 años han modificado de manera profunda las características del paisaje (León et al. 1984; Herrera et al. 2009). La implantación de cultivos comunes a cualquier región templado-húmeda produjo el reemplazo de la vegetación autóctona dominante (Laterra et al. 1998, 2003; Herrera et al. 2009). Esta región posee la matriz agrícola más intensivamente explotada de toda Sudamérica, y presenta un grado elevado de alteración y fragmentación (Viglizzo et al. 2006). El girasol (Helianthus annuus) es un cultivo muy importante dentro de la región Pampeana, y de la Argentina en general, siendo nuestro país uno de los líderes mundiales en su producción. Este cultivo requiere de una polinización mediada por animales para maximizar su productividad (Free 1993). En nuestra región, este cultivo es visitado y polinizado por una gran diversidad de insectos, siendo la abeja melífera el visitante floral dominante (Torretta et al. 2010). Los ambientes ruderales, como las franjas sin cultivar a lo largo de los alambrados, los 
bordes de caminos, arroyos y cuerpos de agua albergan, en algunos casos, una gran diversidad de plantas nativas y endémicas, así como malezas introducidas cuya expansión es promovida por actividades antrópicas como la agricultura, el pastoreo y el fuego. En particular, los bordes de los caminos rurales linderos a los cultivos, podrían proveer hábitats para la nidificación, así como recursos alimenticios para una gran diversidad de polinizadores (Torretta \& Poggio 2013).

En este trabajo ponemos a prueba la hipótesis que la diversidad floral de los bordes de caminos rurales tiene un efecto positivo sobre la riqueza de los visitantes florales en general, y sobre la abundancia de los visitantes florales nativos y de Apis mellifera en particular, que se encuentran en los cultivos de girasol aledaños. Si bien ambos grupos de polinizadores, nativos y $A$. mellifera, pueden utilizar estos hábitats marginales como fuentes de refugio y alimento, esperamos una mayor influencia de la diversidad de recursos a escala local sobre los polinizadores nativos que sobre $A$. mellifera. Esto se debería a las diferencias que presentan ambos grupos de polinizadores en la selección de sitios de nidificación y en sus áreas de forrajeo. Por un lado, los polinizadores nativos, que son en su mayoría abejas de vida solitaria, utilizan para nidificar el suelo, tallos y restos de madera (ver Torretta et al. 2012; Torretta \& Poggio 2013), características que se encuentran frecuentemente en bordes de cultivos. Por otro lado, las abejas melíferas requieren grandes cavidades para formar sus nidos, utilizando de manera preferencial huecos en árboles o grandes agujeros entre rocas o en el suelo (ver Ratnieks et al. 1991), características poco frecuentes en bordes de cultivos. A su vez, $A$. mellifera presenta áreas de forrajeo de varios kilómetros de radio, mientras que la gran mayoría de los polinizadores nativos presentan áreas de forrajeo más restringidas (Beekman \& Ratnieks 2000; Gathmann \& Tscharntke 2002).

\section{MATERIALES y MÉTODOS}

\section{Área de estudio}

El área de estudio se encuentra dentro del Distrito Austral de la región Pampeana (Cabrera 1976), en los partidos de Balcarce y Lobería, ubicados en el sudeste de la Provincia de Buenos Aires, República Argentina. Los veranos son calurosos, con una media de temperatura para el mes de enero de $20.8{ }^{\circ} \mathrm{C}$; los inviernos son templados, con una media de temperatura para el mes de julio de $5.2{ }^{\circ} \mathrm{C}$. La precipitación media anuales es de $~ 800 \mathrm{~mm}$, distribuidos en forma más o menos uniforme a lo largo del año (Burgos \& Vidal 1951). En esta zona, alrededor del 90\% del área está representado por una matriz agrícolo-ganadera donde se cultiva girasol, trigo, maíz, papa y soja, entre otros. El $10 \%$ restante está representada por las sierras pertenecientes al sistema de Tandilia, que conservan gran parte de la vegetación característica de la zona (ver Sabatino et al. 2010; Aizen et al. 2012). El uso extensivo e intensivo de esta región de la llanura pampeana ha generado un grado elevado de alteración y fragmentación del paisaje, donde los ambientes ruderales, en particular los bordes de caminos, representan uno de los pocos hábitats semi-naturales dentro de esta matriz. Los bordes de caminos rurales suelen tener entre 3 y $7 \mathrm{~m}$ de ancho $\mathrm{y}$, además de presentar malezas de origen exótico, también preservan algunas especies de plantas nativas. Las dimensiones de los lotes de girasol estudiados oscilan entre 20 y 50 ha, y fueron cultivados con diferentes clases de híbridos. La apicultura es una actividad económica importante en la zona que se desarrolla con variedades europeas de la abeja melífera, principalmente para la producción de miel.

\section{Muestreos}

El trabajo de campo se llevó a cabo en la temporada de floración del girasol 2008-2009, durante los meses de diciembre, enero y febrero. Se muestrearon un total de 17 lotes de girasol, en los cuales uno de sus bordes se encontraba lindante a un camino rural; en ninguno de ellos se encontraron colmenas a menos de $1.5 \mathrm{~km}$ a la redonda. Las distancias entre los diferentes lotes estudiados variaron en el rango de los 3 a $10 \mathrm{~km}$, lo cual excede las distancias de vuelo esperadas para la mayoría de los taxa de visitantes florales (Gathmann \& Tscharntke 2002; Klein et al. 2004; Greenleaf et al. 2007). En los pocos casos en que las distancias fueron $<3 \mathrm{~km}$, dichos lotes estuvieron caracterizados por cultivos de girasol con una fenología floral no superpuesta, por lo que cada lote estudiado puede ser considerado como una repetición independiente en términos del ensamble de visitantes florales. En cada lote se realizaron 20 censos, en cada uno de los cuales se observaron entre 7 y 15 capítulos florales durante 10 minutos, contando e identificando el número de visitantes florales que contactaban los órganos sexuales de las flores, así como el número de inflorescencias visitadas por cada insecto. Las observaciones se realizaron durante el pico de floración de cada lote; se llevaron a cabo 10 censos por la mañana (entre las 9 y $12 \mathrm{~h}$ ) y 10 por la tarde (entre las 15 y 18 h), durante dos días consecutivos. Más detalles del diseño experimental utilizado en el muestreo de insectos en los cultivos de girasol son provistos en Sáez et al. (2012).

Con el fin de estimar la diversidad floral y de polinizadores del borde del camino rural lindero al cultivo, se estableció una transecta de $100 \mathrm{~m}$ de largo en la que se registró el número de individuos de cada una de las especies de plantas entomófilas 
en floración. Luego, se caminó de nuevo la transecta a paso constante durante un período de 10 minutos y se contaron e identificaron todos los insectos que contactaban las partes reproductivas de las flores abiertas, a una distancia de $1 \mathrm{~m}$ a cada lado del observador (Pollard 1977). La determinación de las especies de plantas y visitantes florales se realizó mediante colecciones de referencia y con la ayuda de especialistas del museo de Ciencias Naturales Lorenzo Scaglia y del Museo Argentino de Ciencias Naturales "Bernardino Rivadavia" (ver agradecimientos). Los visitantes florales que no pudieron ser identificados hasta el nivel de especie fueron clasificados como morfoespecies.

\section{Análisis de datos}

Para cada borde de camino rural lindero al cultivo de girasol estimamos la diversidad floral y de polinizadores presentes mediante el índice de Shannon (H') (Shannon \& Weaver 1949). Mediante el uso de una correlación de Pearson evaluamos la relación entre la diversidad floral del borde y la diversidad de polinizadores presentes en dichos ambientes.

Posteriormente, evaluamos de manera diferencial el efecto de la diversidad floral del borde de cada lote sobre: a) la riqueza de visitantes florales, b) número de visitas de insectos nativos $\mathrm{y}, \mathrm{c}$ ) número de visitas de $A$. mellifera a las inflorescencias de girasol. Para ello se realizaron regresiones jerárquicas generalizadas utilizando la función lmer del paquete lme4 (Bates \& Maechler 2009) ya que nuestro diseño de muestreo estuvo caracterizado por una estructura anidada, que incluye varios censos por lote pero un sólo valor de diversidad de borde para cada lote (pseudorepetición), así como errores residuales no-normales (Tabla 1). El lote se incorporó al modelo como factor aleatorio, mientras que la diversidad floral del borde como factor fijo (Crawley 2007). En los modelos se asumió una distribución del error de Poisson, dado que la riqueza, así como el número de visitas de polinizadores, son variables discretas cuyo límite teórico máximo puede ser asumido como infinito (Crawley 2007). Esta distribución utiliza la función de enlace log, relacionada a la no-normalidad del error (Crawley 2007). Debido a que el número de inflorescencias no fue constante en cada uno de los censos de muestreo, en los análisis del número de visitas este factor fue incluido como una variable "offset" (i.e., variable que sabemos afecta a "y" pero no es de interés conocer la magnitud de su efecto) en el modelo (Gelman \& Hill 2007). Todos los análisis se llevaron a cabo con el software estadístico $\mathrm{R}$ versión 2.13.1 (R Development Core Team 2005) y se usó el método de máxima verosimilitud para la estimación de los parámetros (Crawley 2007). Finalmente, se utilizó la prueba de cociente de verosimilitud (Lehmann \& Romano 1986) para evaluar la existencia de diferencias significativas entre la verosimilitud del modelo nulo (sin variables explicativas) y la de los modelos que presentaban nuestra variable de interés (Tabla 1).

\section{Resultados}

Registramos un total de 2615 visitas en las inflorescencias de los cultivos de girasol. Todos los visitantes florales fueron insectos, con la excepción de una única visita realizada por el "Picaflor verde común", Chlorostilbon aureoventris. De los visitantes florales, $A$. mellifera (Orden Hymenoptera) fue el más abundante ya que realizó $294 \%$ del total de las visitas y fue observada en $97.9 \%$ de todos los censos. Los insectos nativos realizaron el $6 \%$ restante del total de las visitas y fueron observados en $35.8 \%$ de todos los censos. De los visitantes florales silvestres, los más representativos fueron otros Hymenoptera (1.7\% del total de las visitas), seguido por Coleoptera $(1.4 \%)$, y finalmente Diptera, Hemiptera y Lepidoptera, con porcentajes menores al $1 \%$ en la tasa de visita.

En los bordes de caminos linderos a los cultivos de girasol se observaron un total de 20 especies de plantas entomófilas; entre las más conspicuas se encontraron las exóticas Carduus acanthoides y Conium maculatum, las nativas Celtis ehrenbergiana, Verbena bonaerensis y Solanum sublobatum y las endémicas Eryngium horridum y Colletia paradoxa. Las especies de mayor abundancia en estos ambientes fueron Eryngium horridum y Carduus acanthoides, representando 51.4 y $20.2 \%$ de la abundancia

Tabla 1. Modelos lineales mixtos generalizados aplicados a las diferentes variables dependientes y sus correspondientes test de cociente de verosimilitud entre el modelo nulo y el modelo con la variable independiente diversidad del borde, medida con el Índice de Shannon.

Table 1. Generalized lineal mixed models applied to the different dependent variables and the likelihood ratio test between the null model and the model with edge diversity, measured with Shannon-Index.

\begin{tabular}{|c|c|c|c|c|c|c|c|}
\hline $\begin{array}{l}\text { Variable } \\
\text { dependiente }\end{array}$ & Unidades & $\begin{array}{c}\text { Distribución } \\
\text { del error }\end{array}$ & $\begin{array}{c}\text { Función de } \\
\text { enlace }\end{array}$ & $\begin{array}{l}\mathrm{N}^{\circ} \text { de } \\
\text { Grupos }\end{array}$ & $\mathrm{N}^{\mathrm{o}}$ de Obs. & $\Delta \mathrm{AIC}$ & ChiQ \\
\hline Riqueza de especies & No. Taxa por Censo & Poisson & $\log$ & 17 & 291 & -8 & $11.63^{* *}$ \\
\hline Visitas nativos & $\begin{array}{l}\text { No. Visitas } \times \text { inflorescencia } \\
{ }^{1} \times 10 \text { minutos }^{-1}\end{array}$ & Poisson & $\log$ & 17 & 291 & -10 & $12.04^{* * *}$ \\
\hline Visitas A. mellifera & $\begin{array}{l}\text { No. Visitas } \times \text { inflorescencia } \\
{ }^{1} \times 10 \text { minutos }^{-1}\end{array}$ & Poisson & $\log$ & 17 & 291 & 1 & 0.75 \\
\hline
\end{tabular}

Obs.=Observaciones; ${ }^{*} P<0.05,{ }^{* *} P<0.01,{ }^{* * *} P<0.001$ 


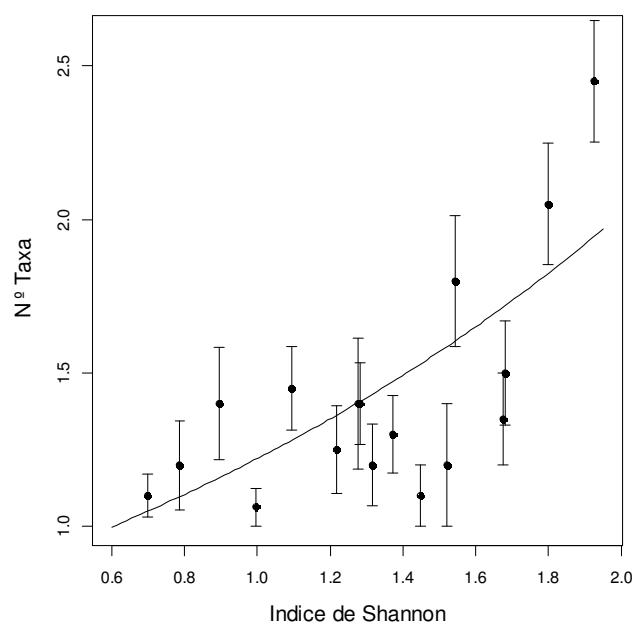

Figura 1. Efecto de la diversidad floral del borde sobre la riqueza de visitantes florales en los cultivos de girasol. En la figura se muestran las medias y desvíos estándar del número de taxones de insectos observados por censo que visitaron las inflorescencias del girasol de los 17 lotes en relación con la diversidad floral del borde lindero al cultivo, medida con el índice de Shannon. La línea representa la curva de regresión estimada por el modelo jerárquico de la riqueza de visitantes florales en función del índice de Shannon (Riqueza $a_{[i]}=a_{j}+\beta_{i} \times$ Diversidad $\left._{j}+\varepsilon_{i i]}\right)$, donde $\mathrm{j}$ representa a cada lote $\left(n_{i}=17\right)$, e i cada censo realizado $\left(n_{i}=291\right)$

Figure 1. Effects of the edge floral diversity on flowervisitors richness in sunflower crops. In this figure are shown the mean and standar deviation of number of insects taxa that visit the sunflower inflorescences per census on the 17 fields related to field-edge floral diversity, measured with Shannon Index. Line represent the regression curve estimated by the hierachical model of flower-visitiors richness vs. the Shannon Index (Richn ess $_{\mathrm{j}[\mathrm{il}}=\mathrm{a}_{\mathrm{i}}+\beta_{\mathrm{i}} \times$ Diversity $\left._{\mathrm{j}}+\varepsilon_{\mathrm{i}[\mathrm{i}}\right)$, where $\mathrm{j}$ represents each field $\left(n_{j}=17\right)$, and i each census $\left(n_{i}=291\right)$.

total y habiendo sido observadas en el $65 \mathrm{y}$ $94 \%$ de todos los censos, respectivamente. La riqueza de especies de plantas entomófilas en estos sitios fue muy variable; en los bordes menos diversos se observaron sólo tres especies, con una dominancia clara de plantas exóticas (principalmente Carduus acanthoides), y entre ocho y nueve especies en los más diversos, con abundancias relativas más equitativas. En nuestrosistema deestudioelíndicedediversidad de Shannon para las especies de plantas varió entre 0.69 (para los bordes menos diversos) y 1.92 (para los bordes más diversos).

Los visitantes florales más representativos en los bordes de cultivos fueron los Diptera ( $43 \%$ del total de la abundancia), seguidos por los Coleoptera (29\%) e Hymenoptera (27\%); A. mellifera fue la especie de visitante floral más abundante de los himenópteros ya que contabilizó el 17\% de la abundancia total. La riqueza de especies de visitantes florales varió entre 4 taxones para los bordes menos diversos, y 11 para los más diversos, mientras la diversidad estimada por el índice de Shannon lo hizo entre 0.80 y 2.2. Nuestros resultados mostraron que la diversidad de los visitantes florales presentes en el borde del cultivo estuvieron correlacionados significativamente con la diversidad floral de dicho borde $(r=0.68, n=17, P=0.002)$, por lo que bordes de cultivo con mayor diversidad floral presentarían una mayor diversidad de polinizadores.

El modelo que evalúa la variación en la riqueza de visitantes florales presentes en el lote de girasol en función de la diversidad floral del borde mostró un valor de AIC significativamente menor que el modelo nulo (Tabla 1). Los cultivos aledaños a los bordes con los mayores niveles de diversidad floral $\left(\mathrm{H}^{\prime}=1.92\right)$ presentaron una riqueza de visitantes florales en promedio dos veces mayor que aquellos cultivos rodeados por los bordes menos diversos $(\mathrm{H}=0.69)$, decreciendo de un total de dos a un taxa por censo $(\beta=0.50, \mathrm{SE}=0.14, P<0.001$; Figura 1$)$. A su vez, el modelo que evalúa la variación en

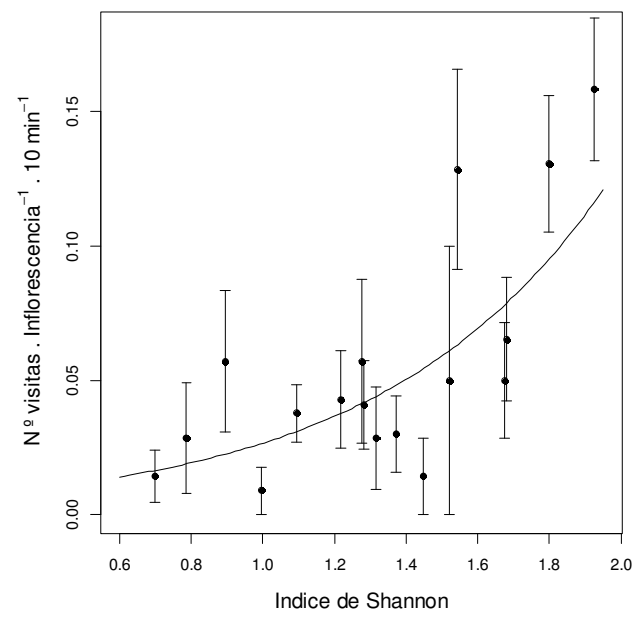

Figura 2. Efecto de la diversidad floral del borde sobre la frecuencia de visita de insectos nativos en los cultivos de girasol. En el gráfico se muestran las medias y desvíos estándar de la frecuencia de visita de insectos nativos a las inflorescencias de girasol de los17 lotes en relación con la diversidad floral del borde lindero al cultivo, medida con el índice de Shannon. La línea representa la curva de regresión estimada por el modelo jerárquico del número de visitas de insectos nativos en función del índice de Shannon $\left(\right.$ Visita $_{\mathrm{jil}}=\mathrm{a}_{\mathrm{i}}+\beta_{\mathrm{j}} \times$ Diversidad $\left._{\mathrm{i}}+\varepsilon_{\mathrm{iji}}\right)$, donde $\mathrm{j}$ representa a cada lote $\left(n_{j}=17\right)$, e i cada censo realizado $\left(n_{j}=291\right)$.

Figure 2. Effects of the edge floral diversity on native insects visit frequency in sunflower crops. In this figure are shown the mean and standar deviation of native insects visit frequency to sunflower inflorescences on the 17 fields related to field-edge floral diversity, measured with Shannon Index. Line represent the regression curve estimated by the hierachical model of native insects visit frequency $v$ s. the Shannon Index $\left(\right.$ Visit $_{i[1]}=a_{i}+\beta_{j} \times$ Diversity $\left._{i}+\varepsilon_{i[i]}\right)$, where $j$ represents each field $\left(n_{j}=17\right)$, and i each census $\left(n_{i}=291\right)$. 
la frecuencia de visitas de insectos nativos a las inflorescencias del cultivo de girasol en función de la diversidad floral del borde también mostró un valor de AIC significativamente menor que el modelo nulo (Tabla 1). Las inflorescencias de cultivos linderos a bordes con los mayores niveles de diversidad tuvieron en promedio diez veces más visitas por insectos nativos que aquellos cultivos rodeados por bordes con los menores niveles de diversidad, decreciendo de 0.1 a 0.01 visitas $\times$ inflorescencia ${ }^{-1} \times 10$ minutos $^{-1}$ $(\beta=1.59, \mathrm{SE}=0.35, P<0.001$; Figura 2$)$. Finalmente, el modelo que evalúa la variación en la frecuencia de visitas de $A$. mellifera a las inflorescencias del cultivo de girasol en función de la diversidad floral del borde, no mostró un valor de AIC significativamente menor que el modelo nulo (Tabla 1). La diversidad floral del borde lindero al cultivo no mostró una relación significativa con la frecuencia de visitas de $A$. mellifera a las inflorescencias del girasol $(\beta=-0.32, \mathrm{SE}=0.37$, $P=0.38$; Figura 3 ). Esto sugiere que la variación en la tasa de visitas de esta especie dominante no puede ser explicada por la diversidad floral presente en el borde del cultivo.

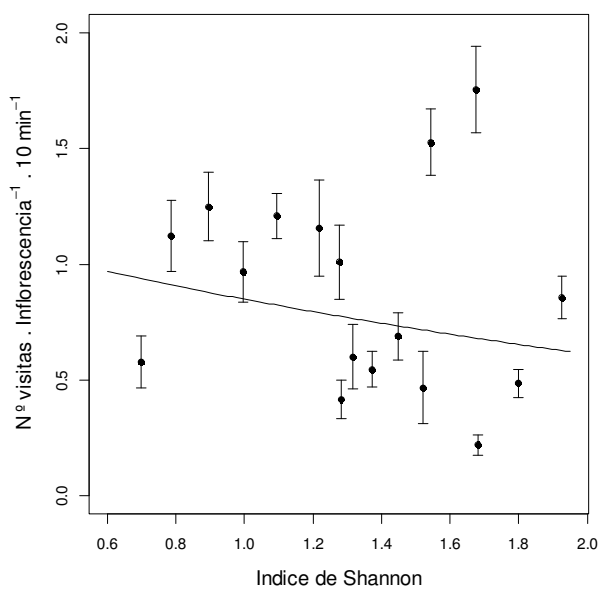

Figura 3. Efecto de la diversidad floral del borde sobre la frecuencia de visita de $A$. mellifera en los cultivos de girasol. En el gráfico se muestran las medias y desvíos estándar de la frecuencia de visita de $A$. mellifera a las inflorescencias de girasol de los17 lotes en relación con la diversidad floral del borde lindero al cultivo, medida con el índice de Shannon. La línea representa la curva de regresión estimada por el modelo jerárquico del número de visitas de $A$. mellifera en función del índice de Shannon $\left(\right.$ Visita $_{\mathrm{j}[\mathrm{i}}=\mathrm{a}_{\mathrm{j}}+\beta_{\mathrm{j}} \times$ Diversidad $\left._{\mathrm{j}}+\varepsilon_{\mathrm{j}[\mathrm{i}]}\right)$, donde j representa a cada lote $\left(n_{j}=17\right)$, e i cada censo realizado $\left(n_{i}=291\right)$.

Figure 3. Effects of the edge floral diversity on A. mellifera visit frequency in sunflower crops. In this figure are shown the mean and standar deviation of $A$. mellifera visit frequency to sunflower inflorescences on the 17 fields related to fieldedge floral diversity, measured with Shannon Index. Line represent the regression curve estimated by the hierachical model of $A$. mellifera visit frequency $v$ s. the Shannon Index $\left(V_{i s i t}{ }_{i[j]}=a_{j}+\beta_{j} \times\right.$ Diversity $\left._{j}+\varepsilon_{\mathrm{j}[\mathrm{ij}}\right)$, where $\mathrm{j}$ represents each field $\left(n_{j}=17\right)$, and i each census $\left(n_{i}=291\right)$.

\section{DISCUSIÓN}

Nuestros resultados sustentan la hipótesis de que la diversidad floral presente en el borde de los caminos rurales afecta la riqueza y abundancia de los visitantes florales nativos presentesenelcultivodegirasolaledaño.Sibien algunos autores han enfatizado la importancia de los remanentes de hábitats naturales y seminaturales por la gran diversidad de especies que albergan (Hendrickx et al. 2007; Billeter et al. 2008) y como fuente de diferentes servicios ecosistémicos en el paisaje agrícola, incluido el de polinización (Costanza et al. 1997; Kremen et al. 2004; Chacoff \& Aizen 2006; Carvalheiro et al. 2011), nuestro trabajo aporta información novedosa para el área de estudio, en donde los ambientes ruderales son escasamente valorados como proveedores de servicios ecosistémicos en los agroecosistemas (sin embargo, ver Torretta \& Poggio 2013).

Los insectos nativos no fueron los visitantes florales dominantes en el presente estudio, ya que realizaron el 6\% de las visitas totales. Sin embargo,Garibaldietal.(2013)handemostrado la gran importancia que tiene la presencia de ensambles diversos de polinizadores en la productividad de una gran diversidad de cultivos, incluido el girasol. Incluso aunque su abundancia total sea baja, estos ensambles diversos de polinizadores, mayormente compuestos por especies nativas, pueden cumplir un rol aún más importante que el de A. mellifera en la polinización y producción de semillas y/o frutos (Klein et al. 2007; Garibaldi et al. 2013). De hecho, para el caso específico del girasol, Greenleaf \& Kremen (2006a) demostraron que las abejas nativas pueden tener un efecto indirecto sobre la polinización al interferir con las visitas de A. mellifera. Este tipo de competencia incrementa la distancia entre flores visitadas consecutivamente por las abejas melíferas, y de esta forma aumenta la tasa de alogamia. Nuestros resultados mostraron que tanto la riqueza como la frecuencia de visita de los visitantes florales nativos a las inflorescencias del girasol se ven influenciadas de forma positiva por la diversidad floral presente en el borde de los caminos rurales linderos al cultivo. Por lo tanto, la diversidad de estos bordes sería uno de los factores que determinaría cuan abundante y diverso es el ensamble de polinizadores que pueden albergar y, en consecuencia, podría afectar la calidad del servicio de polinización a los cultivos aledaños.

Los bordes de caminos, y de cultivos en general, representan uno de los pocos 
hábitats que preservan, aún hoy, parte de la vegetación autóctona de la zona. Sin embargo, la explotación de estos ambientes con fines agropecuarios (e.g., siembra y pastoreo), así como quemas y aplicaciones de herbicidas casuales y/o intencionales, modifica la estructura de la comunidad de plantas, y por consiguiente, la disponibilidad de recursos para los organismos de niveles tróficos superiores, conduciendo a una pérdida paulatina de la biodiversidad y a una simplificación del paisaje cada vez mayor. La extensión y características de los ambientes naturales y semi-naturales que se encuentran presentes en los agroecosistemas dependen del tipo de manejo que se lleve a cabo (Burdel et al. 2002; Gibson et al. 2007). Con el fin de aumentar y/o preservar la biodiversidad y los servicios ecosistémicos que estos brindan, en algunas regiones del mundo se establecieron reglamentaciones ambientales de obligación legal, o incentivos financieros, con el objetivo de preservar estos ambientes, entre ellos los márgenes de cultivos (Birch 2005; Radley et al. 2005). Sin embargo, la mayor parte de la región pampeana no presenta regulaciones en este ámbito, por lo que el uso y manejo que se realicen sobre los ambientes naturales como semi-naturales dependen en gran medida de las decisiones tomadas por el agricultor.

La abeja melífera representa claramente el visitante floral más abundante, y contribuye con más del $94 \%$ en el total de las visitas a los cultivos de girasol en el sudeste de la Provincia de Buenos Aires. Resultados similares fueron reportados por Torretta et al. (2010), quien relevó ocho áreas agrícolas en la Argentina, incluida la región Pampeana. Esta dependencia casi exclusiva de la polinización por $A$. mellifera observada en los cultivos de girasol, podría tener serias consecuencias debido a que en los últimos seis años el número de colmenas ubicadas en las Provincia de Buenos Aires, Córdoba, Santa Fe y La Pampa disminuyó aproximadamente en un 50\% (Fernando 2010). Las principales causas dela pérdida en las poblaciones de abejas domésticas están asociadas al uso masivo e indiscriminado de herbicidas como el glifosato, relacionado a su vez a la expansión de la soja transgénica en los últimos años (Fernando 2010). Esto ha generado una creciente y constante pérdida de la diversidad vegetal, así como interrupciones temporales en la provisión de recursos florales, críticos para la nutrición de las abejas en épocas donde los cultivos no se encuentran en flor (Fernando 2010). Problemas similares ya se ha observado en otras regiones del mundo como en los Estados Unidos (NRC 2007) y Europa
(Winfree et al. 2009), en donde enfermedades infecciosas y el uso inadecuado de pesticidas han producido una declinación en las poblaciones de abejas melíferas (Kluser \& Peduzzi 2007).

Nuestros resultados no aportan ninguna evidencia de que la frecuencias de visita de A. mellifera en los lotes de girasol esté relacionada con la diversidad floral presente en el borde del cultivo, posiblemente debido a que la variación en la tasa de visita de este polinizador se encuentra influenciada por otros factores que responden a la escala de paisaje. Anteriormente encontramos que la distancia de los lotes de girasol a las sierras, grandes extensiones de ambientes naturales presentes en el área, se relacionaba positivamente con la abundancia de la abeja melífera en los cultivos (Sáez et al. 2012). Por otro lado, no encontramos evidencia de que la diversidad floral de los bordes de los cultivos esté asociada con su proximidad a las sierras ( $r=-0.10, n=17, P=0.69)$. En consecuencia, concluimos que la abundancia y tasas de visitas de $A$. mellifera estarían determinadas por factores que varían más a una escala regional (Beekman \& Ratnieks 2000; Steffan-Dewenter \& Kuhn 2003) que a escala local, lo cual podría ser explicado por el radio de forrajeo de varios kilómetros que exhiben los individuos de esta especie. Si bien no encontramos relación entre la abundancia de $A$. mellifera en el cultivo y la diversidad del borde, pudimos observar que las abejas melíferas frecuentemente visitan las flores de los bordes de caminos rurales en busca de recursos (e.g., néctar y/o polen). Esto sugiere que dichos ambientes brindan recursos complementarios y suplementarios a la dieta de la abeja melífera, lo que quizás favorezca su supervivencia en épocas del año donde la oferta de recursos por parte de los cultivos sea escasa o nula. Para poder verificarlo, sería oportuno la realización de otros trabajos donde se analice la abundancia de $A$. mellifera en los bordes de caminos rurales, en función de la disponibilidad de cultivos en el área en diferentes momentos del año.

\section{Implicancias en la conservación y en la producción agrícola}

Nuestros resultados apoyan la hipótesis de que el grado de preservación de los bordes de caminos rurales determina que tan diverso y abundante es el elenco de polinizadores presentes en los cultivos de girasol aledaños. La diversidad floral presente en estos ambientes semi-naturales, no sólo puede robustecer el reservorio de polinizadores disponible para 
aquellos cultivos dependientes de ellos, sino que también, junto con los ambientes naturales remanentes, brindan recursos que contribuyen a sostener el servicio de polinización. Por tales motivos se hace necesario un manejo sustentable del espacio rural, así como una adecuada planificación de la actividad agrícola, con el objetivo de promover la biodiversidad y sustentabilidad de los agroecosistemas.

Agradecimientos: Agradecemos especialmente a C. Morales, N. Chacoff y L. Herrera por sus útiles comentarios y sugerencias que han realizado a nuestro manuscrito. A.J. Farina y R. González Vaquero por su ayuda en la determinación de los insectos. El trabajo fue financiado por el Instituto Nacional de Tecnología Agropecuaria, Balcarce (PNECO 1302), el Consejo Nacional de Investigaciones Científicas y Técnicas (PIP 01623), el Fondo Nacional de Investigación (PICT 01300) y por la Universidad Nacional del Comahue (B152/04).

\section{BibLIOGRAFíA}

Aizen, MA; M Sabatino \& JM Tylianakis. 2012 Specialization and rarity predict non-random loss of interactions from mutualist networks. Science, 335: 1486-1489.

Altieri, MA. 1999. The ecological role of biodiversity in agroecosystems. Agriculture Ecosyst. Environ., 74: 19-31.

BATES, D \& M MAECHLER. 2009. Ime4: Linear Mixed-Effects Models Using S4 Classes. R package version 0.999375-32. http: / /CRAN.R-project.org/Package=lme4.

BEEKMAN, M \& FLW RATNIEKS. 2000. Long-range foraging by the honeybee, Apis mellifera L. Funct. Ecol., 14:490-496.

Billeter, R; J Litra; D Bailey; R Bugter; P Arens; ET Al. 2008. Indicators for biodiversity in agricultural landscapes: a pan-European study. J. Appl. Ecol., 45:141-150.

$\mathrm{BIRCH}$, S. 2005. Farming for wildlife. BBC Wildlife, Autumn, 2005:44-50.

BRONSTEIN, JL. 1995. The plant-pollinator landscape. Pp. 256288 en: Hansson, L; L Fahrig \& G. Merriam (eds.). Mosaic landscapes and ecological processes. Chapman and Hall.

Burgos, JJ \& AL Vidal. 1951. Los climas de la República Argentina, según la nueva clasificación de Thornthwaite. Meteoros, 1:3-32.

CABrerA, AL. 1976. Regiones fitogeográficas argentinas. Pp. 1-85 en: Kugler, WF (ed.). Enciclopedia Argentina de Agricultura y Jardinería. II, ACME, Buenos Aires.

Carvalheiro, LG; R Veldtman; AG Shenkute; GB Tesfay; CW PIRK; ET AL. 2011. Natural and within-farmland biodiversity enhances crop productivity. Ecol. Lett., 14:251-259.

Chacoff, NP \& MA Aizen. 2006. Edge effects on flowervisiting insects in grapefruit plantations bordering premontane subtropical forest. J. Appl. Ecol., 43:18-27.

Chacoff, NP; MA Aizen \& V Aschero. 2008. Proximity to forest edge does not affect crop production despite pollen limitation. Proc. R. Soc. Lond. B, 275:907-913.

Clough, Y; A Kruess \& T TscharntKe. 2007. Local and landscape factors in differently managed arable fields affect the insect herbivore community of a non-crop plant species. J. Appl. Ecol., 44:22-28.

Costanza, R; RD'Arge; RDe Groot; S FARber; M Grasso; ET AL. 1997. The value of the world's ecosystem services, and natural capital. Nature, 387:253-260.

CRAWLeY, MJ. 2007. The R Book. Imperial College London at Silwood Park, UK. Pp. 527-528.

FERNANDO, LE. 2010. Ley de incentivo a la diversificación productiva agropecuaria. Espacio apícola, 92:22-38.

GATHMANN, A \& T TSCHARNTKE. 2002. Foraging ranges of solitary bees. J. Anim. Ecol., 71:757-764.

FREE, JB. 1993. Insect pollination of crops. Academic, San Diego.

Garibaldi, LA; I STEFFAn-DeWEnter; R Winfree; MA Aizen; R BOMMARCO; ET AL. 2013. Wild pollinators enhance fruit set of crops regardless of honey bee abundance. Science, 339:1608-1611. DOI: 10.1126/science.1230200.

Gelman, A \& J Hill. 2007. Data analysis using regression and multilevel/hierarchical models. Cambridge Universty Press. Pp. 109-116.

GHazoul, J. 2006. Floral diversity and the facilitation of pollination. J. Ecol., 94:295-304.

Gibson, RH; S PEARCE; RJ MorRIs; WO SYMONDSON \& J Меммотт. 2007. Plant diversity and land use under organic and conventional agriculture: a whole-farm approach. J. Appl. Ecol., 44:792-803.

Greenleaf, SS \& C Kremen. 2006a. Wild bees enhance honey bees' pollination of hybrid sunflower. Proc. Nat. Acad. Sci., 103:13890-13895.

Greenleaf, SS \& C Kremen. 2006b. Wild bee species increase tomato production and respond diferently to surrounding land use in Northern California. Cons. Biol., 133:81-87.

Greenleaf, SS; MN Williams; R Winfree \& C KREMEN. 2007. Bee foraging ranges and their relationship to body size. Oecología, 153:589-596.

Hendrickx, F; JP Maelfait \& W van Wingerden. 2007. How landscape structure, land-use intensity and habitat diversity affect components of total arthropod diversity in agricultural landscapes. J. Appl. Ecol., 44:340-351.

Herrera, LP; P Laterra; NO Maceira; KD Zelaya \& GA MARTínez. 2009. Fragmentation status of tall-tussock grassland relicts in the flooding Pampa, Argentina. Rangel. Ecol. Manag., 62:73-82.

Klein, AM; I StefFan-Dewenter \& T Tscharntke. 2003a. Fruit set of highland cofee increases with the diversity of pollinating bees. Proc. R. Soc. Lond. B, 270:955-961.

KLeIn, AM; I StefFan-Dewenter \& T TscharntKe. 2003b. Pollination of Coffea canephora in relation to local and regional agroforestry management. J. Appl. Ecol., 40: 837-845.

Klein, AM; I Steffan-Dewenter; T Tscharntke. 2004. Foraging trip duration and density of megachilid bees, eumenid wasps and pompilid wasps in tropical agroforestry systems. J. Anim. Ecol., 73:517-525.

KLeIN, AM; BE VAISSIERE; JH CANE; I STEFFAN-DeWENTER; SA CUNNINGHAM; ET AL. 2007. Importance of pollinators in changing landscape for world crops. Proc. R. Soc. Lon. B., 274:303-313.

Kluser, S \& P Peduzzi. 2007. Global pollinator decline: A literature review. Geneva: UNEP/GRID.

Kremen, C; NM Williams \& RW Thorp. 2002. Crop pollination from native bees at risk from agricultural intensification. Proc. Nat. Acad. Sci., 99:16812-16816.

Kremen, C; NM Williams \& RL Bugg. 2004. The area requirements of an ecosystem service: crop pollination by native bee communities in California. Ecol. Lett., 7: 1109-1119.

KREMEN, C. 2005. Managing ecosystem services: what 
do we need to know about their ecology? Ecol. Lett., 8:468-479.

Kudo, G. 2006. Flowering phenologies of animalpollinated plants: reproductive strategies and agents of selection. Pp. 139-155 en: Barrett SCH \& LD Harder (eds.). Ecology and evolution of owers. Oxford; Oxford University Press.

Laterra, P; OR Vignolio; LG Hidalgo; ON Fernández; MA Cahuepe; et al. 1998. Dinámica de pajonales de paja colorada (Paspalum spp.) manejados con fuego y pastoreo en la Pampa Deprimida Argentina. Ecotrópicos, 11:41-149.

Laterra, P; O Vignolio; P Linares; A Giauinta \& NO MACEIRA. 2003. Cumulative effects of fire on the structure and function of a tussock Pampa grassland. J. Veg. Sci., 14:43-54.

León, RJC; GM Rusch \& M Oesterheld. 1984. Pastizales Pampeanos: impacto Agropecuario. Phytocoenologia, 12:201-218.

Lehmann, EL \& JP Romano. 1986. Testing Statistical Hypotheses. Wiley, New York. National Research Council (NRC). 2007. Status of pollinators in North America. National Academies Press, Washington D.C.

Olesen, JM; J Bascompte; H Elberling \& P Jordano. 2008. Temporal dynamics of a pollination network. Ecol., 89: 1573-1582.

Ollerton, J; R Winfree \& S TarRant. 2011. How many flowering plants are pollinated by animals? Oikos, 120: 321-326.

Pollard, E. 1977. A method for assessing changes in the abundance of butterflies. Biol. Cons., 12:115-134.

Potts, SG; JC Biesmeijer; C Kremen; P Neumann; O SCHWEIGER; ET AL. 2010. Global pollinator declines: trends, impacts and drivers. Trends Ecol. Evol., 25:345-353.

R Development Core Team. 2005. R: A Language and Environment for Statistical Computing. R Foundation for Statistical Computing. Vienna. Austria. ISBN 3900051-07-0. http:/ / www.R-project.org.

Radley, G; JO'Reilly \& A JowitT. 2005. Environmental Stewardship. British Wildlife, 16:257-263.

RATNIEKS, FLW \& MA PIERY. 1991. The natural nest and nest density of the Africanized honey bee (Hymenoptera, Apidae) near Tapachula, Chiapas, Mexico. The Can. Ent., 123:353-359.
RicKETTS, TH. 2004. Tropical forest fragments enhance pollinator activity in nearby coffee crops. Biol. Cons., 18:1262-1271.

Ricketts, TH; J Regetz; I Steffan-Dewenter; SA Cunningham; C KREMEN; ET AL. 2008. Landscape effects on crop pollination services: are there general patterns? Ecol. Lett., 11:499-515.

Sabatino, M; N Maceira \& MA Aizen. 2010. Direct effects of habitat area on interaction diversity in pollination webs. Ecol. Appl., 20:1491-1497.

SÁez, A; M SABatino \& MA Aizen. 2012. Interactive Effects of Large- and Small-Scale Sources of Feral Honey-Bees for Sunflower in the Argentine Pampas. PLoS ONE, 7(1): e30968. doi:10.1371/journal.pone.0030968.

Sala, OE; FS Chapin; JJ Armesto; E Berlow; J Bloomfeld; et AL. 2000. Global Biodiversity Scenario for the year 2100 . Science, 287:1770-1774.

SHANNON, CE \& W WeAver. 1949. The mathematical theory of communication. University Illinois Press, Urban. Pp. 117.

SteFFan-Dewenter I \& A KuHn. 2003. Honeybee foraging in differentially structured landscapes. Proc. R. Soc. Lond. $B, 270: 569-575$.

Torretta, JP; D Medan; AR Alsina \& NH Montaldo. 2010. Visitantes florales diurnos del girasol (Helianthus annuus, Asterales: Asteraceae) en la Argentina. Revista de la Sociedad Entomológica Argentina, 69:17-32.

TorretTA, JP; SP Durante; MG Colombo \& AM Basilio. 2012. Nesting biology of the leafcutting bee Megachile (Pseudocentron) gomphrenoides (Hymenoptera: Megachilidae) in an agro-ecosystem. Apidologie, 43: 624-633.

Torretta, JP \& SL Poggio. 2013. Species diversity of entomophilous plants and flower-visiting insects is sustained in the field margins of sunflower crops. J. Nat. His., 47:139-165.

Viglizzo, EF; FC Frank \& L Carreño. 2005. Situación ambiental en las ecorregiones Pampa y Campos y Malezales. Pp. 263-269 en: Brown, A; U Martínez Ortiz; M Acerbi \& J Corcuera (eds.). La situación ambiental argentina. Fundación Vida Silvestre Argentina, Buenos Aires.

Winfree, R; R Aguilar; DP Vazquez; GLE Buhn \& MA Aizen. 2009. A meta-analysis of bees responses to anthropogenic disturbance. Ecol., 90:2068-2076. 